\title{
Cell Dormancy in Cellular Automata
}

\author{
Mohammad Ali Javaheri Javid and Rene te Boekhorst \\ School of Computer Science \\ Faculty of Engineering and Information Sciences \\ University of Hertfordshire \\ Hatfield, Hertfordshire AL10 9AB \\ United Kingdom \\ javaheri@javaheri.info, r.teboekhorst@herts.ac.uk
}

\begin{abstract}
This paper describes a novel implementation of a two-dimensional Cellular Automaton (CA) by introducing a dormant state. An overview of the use of CA's in the field of Artificial Life reveals that certain crucial aspects of biological realism have been sacrificed in favour of abstraction or have not been considered at all. Conway's famous "Game of Life" model includes certain fundamental aspects of population dynamics, including the transition from living state to dead state. But even the simplest biological system consists of more stages than the binary state in the Game of Life. The aim of this research is to build an extended CA model of natural biological systems by introducing a dormant state and to investigate the effect of dormancy on simple population dynamics.
\end{abstract}

\section{Introduction}

The rapidly growing field of Artificial Life is inspired by biological systems and exploits computer technology to synthesize and simulate common processes and characteristics of living organisms. Artificial Life can be used as powerful tool in a variety of fields like psychology, biology, therapy, robotics, Artificial Intelligence, evolutionary computation, art, etc [1]. An overview of the field reveals that some aspects of biological systems lost in abstraction or simply have not been considered. The famous Game of Life introduced by John Conway resembles the population dynamics by including the transition from living state to dead state. However, observations from developing real biological systems show that they go through more stages than the binary states of the Game of Life. In this paper we consider a more realistic CA model of simple biological systems in the theoretical framework of Artificial Life by including and investigating the effect of dormancy on the dynamics of an artificial population.

\section{Background}

Artificial Life considers living systems as dynamical systems in which life is an emergent rather than an inherent property of those systems. This view departs from 
the classical biological framework which studies living systems by decomposing them from top to down in order to understand the mechanism of life. The top-down approach describes the mechanics of life in terms of complex, hierarchical biochemical system. However, life cannot be understood completely in terms of its parts, Instead, the exhibited properties of living systems are the result of interactions between their components. Artificial Life, by adapting a bottom-up approach, tries to synthesize living systems by combining separate elements or substances to form a coherent whole [2] in a medium like computers or robots. In this approach, the whole is different than the sum of its constituents.

\subsection{Cellular Automaton and the Game of Life}

Cellular Automata (CA) are dynamical systems in which both space and time are represented as discrete units. They have been conceived by von Neumann and Ulam to provide a formal framework for investigating the behaviour of complex, extended systems [3] and to study the process of self-reproduction. Von Neumann was interested in the essence of reproduction and not in any particular, implementation of the process. Thus, he purposely abstracted away all the details of, say, how animals reproduce, and instead concentrated on the simplest mathematical framework that allows information to reproduce [4].

A cellular automaton consists of an array of cells, each of which can be in one of a finite number of possible states (known as finite-state automaton), updated synchronously or asynchronously [see 5] in discrete time steps, according to local interaction rules. The state of a cell at the next time step is determined by the current states of a surrounding neighbourhood of cells [6, 7].

Conway introduced the "Game of Life" as a very simple Boolean CA [8]. But despite its simplicity, the "Game of Life" gives rise to complicated behaviour. The cells in the Game of Life have two states, $\{0,1\}$, which are interpreted as live or dead states.

The rule structure of $\mathrm{CA}$ in the Game of Life is given a Moore Neighbourhood (eight possible neighbours: N, W, S, E, NE, NW, SE and SW of a reference cell):

1. A cell becomes alive if three of its neighbours are alive.

2. A cell remains alive if two or three of its neighbours are alive, otherwise it dies either from exposure (number of neighbours $\leq 1$ ) or from overcrowding (number of neighbours $\geq 4$ ).

\section{Life and Death in Biological and Synthesised Systems}

Any attempt in creating new forms of life in artificial media must have clear idea about the nature of life. Life can be studied at a molecular,- cellular,- organismal-, population - and ecosystem level [9]. Next, one could try to extract key properties of life which occur in all the stated levels. So far, attempts to define life are based on listing such properties. Properties common to many lists include the abilities to replicate, evolve, metabolize, respond to stimuli, and to repair damage [10]. 
Problem arises from a lack of agreement on what should or should not be included in the list [see 11, 12]. The properties that are considered to distinguish a living system from a non-living one are not consistent enough to give an appropriate overview of living systems. This raises the problem that if a system lacks the properties of life, then the system could be either dead or "just not alive". According to our knowledge, now non-living system are not distinguished from dead systems in the literature so far.

\section{Dormancy}

Dormancy is a well-known feature of many biological systems. Biological systems (e.g. bacteria) due to stress conditions (say lack of nutrition) may enter into dormant state with relatively low metabolism and ceased replication. As the environmental condition changes, the system regains its active state properties like high metabolism and reproduction. Dormancy is considered to have survival value in that it helps to endure stressful circumstances. This metabolically inactive but reversible state may occur at different levels. When it involves the whole organism, it is more likely to be periodic like winter hibernation in animals and seasonal dormancy in plants. In some cases dormancy is limited to reproductive organs like seeds and spores. In all cases, the system regains its active state properties once the environmental conditions have become favourable again.

Stress conditions to which many bacteria are being exposed apparently did not call for the evolution of highly sophisticated resistant structures [13]. Bacteria in the dormant state function at very low metabolic rates and do not undergo cell division. When the stress is released, bacteria resume cell division. Thus, the transformation to a dormant state considered here involves adaptive mechanisms but no morphological differentiations such as sporulation $[14,15]$.

\section{Dormancy in Artificial Systems}

Reproduction is a fundamental character of living systems. In von Neumann's initial thought-experiment, artificial reproduction was realized in a universal constructor surrounded by components: given the description of any machine, it will locate the proper parts and re-construct that machine. If given a description of itself, it will construct itself [2].Thus, reproduction can be viewed as self-reconstruction, explained in terms of interactions of simple elements and studied as logical principles independent of its physical realization [16].

Von Neumann's model and Langton's loops assume certain minimal requirements for a system to exhibit self-reconstruction. However, should a system be called "dead" or "not alive" when these requirements are not met? In this paper, and inspired by the existence of dormant stages in biological systems, we would like to extend these notions by introducing an intermediate state in artificial systems. For this purpose, we define a dormant state as a state in which a system fails to exhibit the properties of living systems. However it is able to reverse to a previous state in which 
it is able to display these properties. Translating this definition in the context of a twodimensional CA given a Moore Neighbourhood, a dormant cell is a cell which in a previous time step $(t-1)$ was in the living state $\{1\}$ and in the time $(t)$ it is in the dormant state $\{0.5\}$. Considering the state transition of dormant cells in biological systems, we distinguish the following types of dormancy for cells in a CA:

Dormancy A: This is a static dormancy which maintains its dormant value of $\{0.5\}$ for all time steps.

1. A cell becomes alive if three of its neighbours are alive (reproduction).

2. A cell remains alive if two or three of its neighbours are alive otherwise it enters into a dormant state.

These rules are identical to Conway's Game of Life, the only difference being that cells enter into dormant state (and therefore still occupy space) instead of dying (and hence leave behind an open space).

Dormancy B: This is a type of dormancy which, due to ongoing environmental stress can change a dormant state $\{0.5\}$ into a dead state of $\{0\}$ :

1. An empty cell becomes alive if three of its neighbours are alive (reproduction), otherwise it stays empty

2. A cell stays alive if two or three of its neighbours are alive otherwise it enters into the dormant state (maintenance)

3. A cell remains dormant if less than four of its neighbours are dormant otherwise it dies.

Dormancy C: This includes a phase of dormancy which, due to favourable environmental factors, regains the state of being alive $\{1\}$ :

1. A cell becomes alive if three of its neighbours are alive, otherwise it stays empty.

2. A cell remains alive if two or three of its neighbours are alive otherwise it enters into dormant state.

3. A cell remains dormant if four of its neighbours are dormant and dies if it has more than 4 neighbours.

4. A dormant cell becomes alive if two or three of its neighbours are dormant.

The rule number four retains the situation that due to environmental changes which favour reproduction, dormant cells will be able to regain their live states.

\section{Simulations and Results}

The rule tables of the three systems were implemented in a CA environment with wrap-around Moore neighbourhood to test the effect of a set of initial conditions. The experimental initial states are the "glider" and a rectangular "block" with a perimeter of 14 cells (W). Figure 1 illustrates snapshots from some later stages in the pattern development for dormancy type A starting from a "glider" and figure 2 shows the same when starting with a "block". Figures 3 to 6 show the results of runs for both 
initial configurations for respectively dormancy types B and C. In figures (1 to 6) the final state is a state that the growth of cells continues unless there is no place to grow (Full colour snapshots are available from:http://www.javaheri.info/dormancy.html).

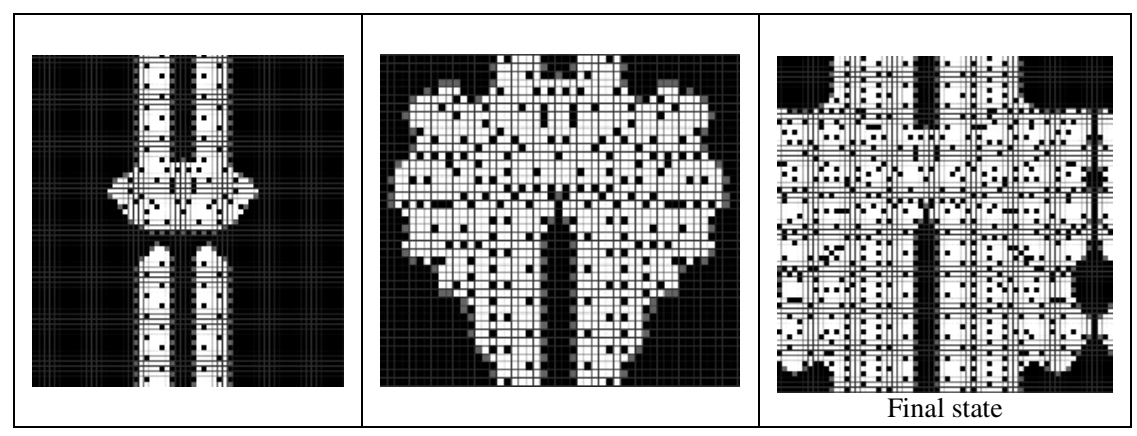

Fig. 1. Development of patterns in dormancy type A for Glider initial state. White = dormant cells, Grey = living cells.

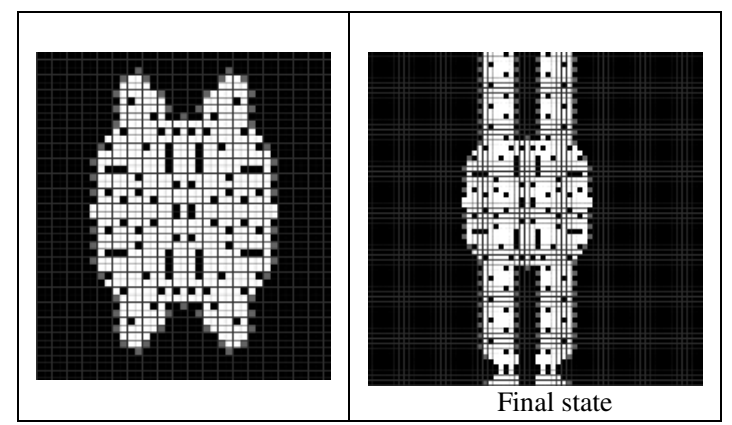

Fig. 2. Development of patterns in dormancy type A for Block initial state

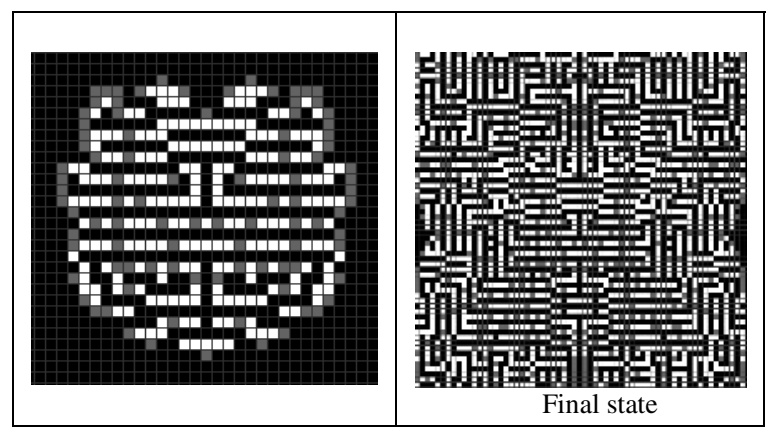

Fig. 3. Development of patterns in dormancy type B for Glider initial state 


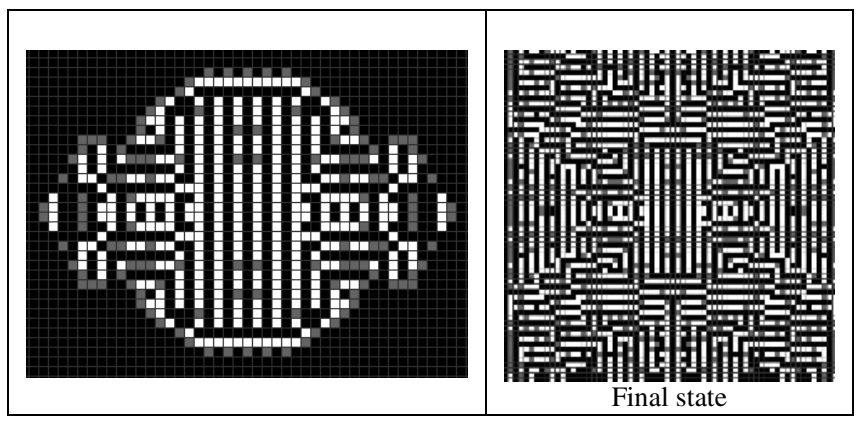

Fig. 4. Development of patterns in dormancy type B for Block initial state

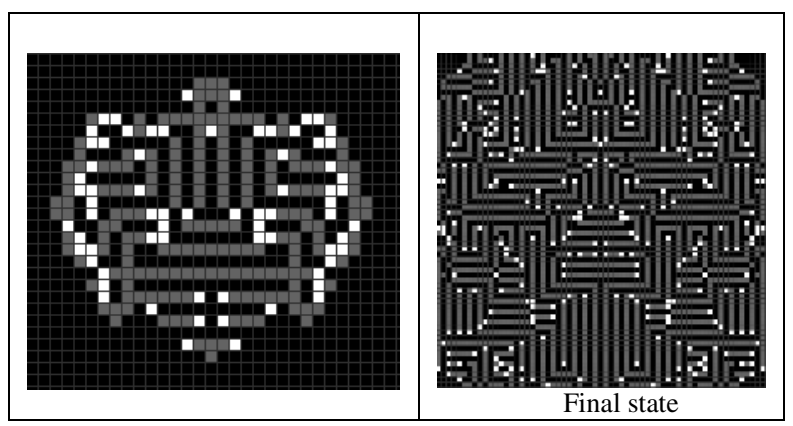

Fig. 5. Development of patterns in dormancy type $\mathrm{C}$ for Glider initial state

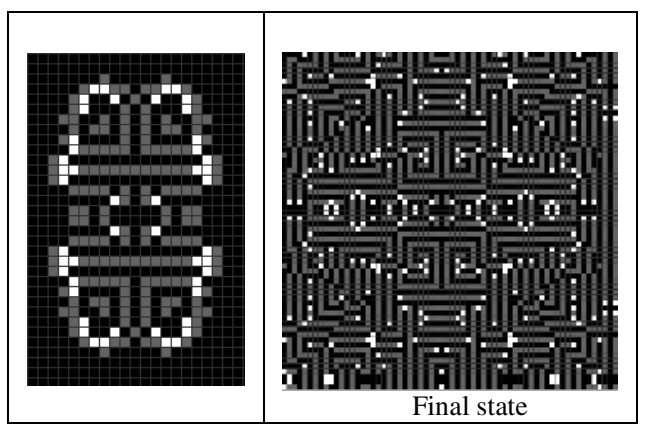

Fig. 6. Development of patterns in dormancy type C for Block initial state

The experiment conducted for ten consecutive time steps and compared with those of the classical Game of Life. Simulations show that from the third time step the Game of Life results in a fixed number of living cells, i.e. an attractor. The development of the systems including dormancy (A, B and C) is very different. Obviously, dormancy affects the population dynamics of artificial cells in a CA environment. Figure 7 shows that the number of living cells is increased in systems that include dormant states, which suggests survival value. 
The initial state plays an important role. If living cells are in close proximity of each other - even if randomly located - their growth continues unless there is no place to grow (final state). We found that for dormancy type A, cell growth occurs at the edges of an area covered by dormant cells (figure 1 and 2). Furthermore, in dormancy type A for Glider initial state, after time step $\{10\}$, the population splits into two parts and only grows upwards (figure 1) which is reminiscent of a kind of directed tissue growth. In a torpid CA environment, when the two parts meet each other, there is explosion of population growth which accelerates the growth of dormant cells. We also noticed that the final steady state for both Glider and Block initial states are symmetric. As the figures $1-6$ show, the final state of all dormancy types produce a symmetric pattern.
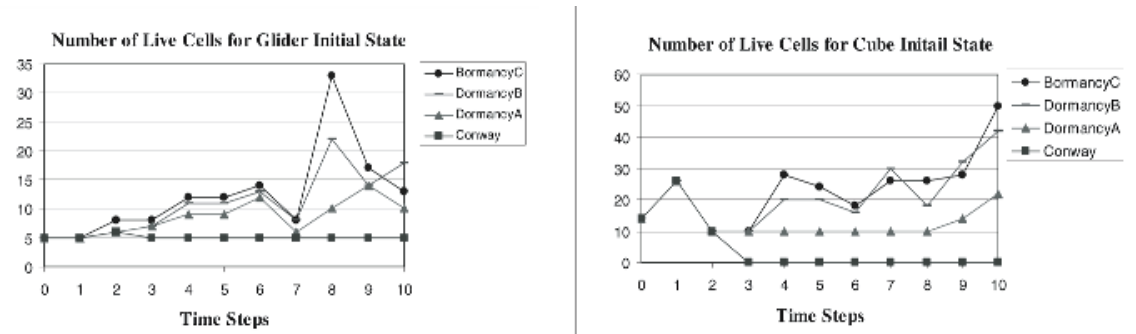

Fig. 7. Dynamics of the number of living cells in different dormancy systems compared to Conway's Game of Life

\section{Conclusions and Further Work}

The Game of Life is a very simple two-dimensional CA which abstracts natural biological systems into two discrete states. Inspired by natural biological systems we implemented a Game of Life with an intermediate dormant state. This led to the development of a model that describes different types. Analysis of the runs showed that the introduction of dormant state significantly affects the population dynamics of living cells. The increased number of living cells in different dormancies suggests that dormancy has survival value for an artificial system in a CA environment. The shape of the patterns depends on the initial configuration and their growth pattern is symmetric. As possible further work for our dormancy models, we suggest investigating whether there is relationship between number of dormant cells and living cells.

\section{References}

1. Langton C. G. and Shimohara K.: Artificial Life V: Proceedings of the Fifth International Workshop on the Synthesis and Simulation of Living systems, The MIT Press/A Bradford Book (1996).

2. Langton, C. G.: Artificial Life, In Langton C.G. (ed.) Artificial Life, Addison-Wesley, (1989) 1- 47.

3. von Neumann, J.: Theory of Self-Reproducing Automata, University of Illinois Press, Illinois. Edited and completed by A. W. Burks (1996). 
4. Flake, G.W.: The computational beauty of Nature, MIT press (1998).

5. Nehaniv, C. L.: Evolution in asynchronous cellular automata, Proceedings of the eighth international conference on artificial life, (2002) 65-73.

6. Wolfram, S.: Universality and complexity in cellular automata. Physica D, Vol. 10 (1984)1-35.

7. Tofoli, T. and Margolus, N.: Cellular Automata Machines, The MIT Press, Cambridge, Massachusetts (1987)

8. Gardner, M.: The fantastic combinations of John Conway's new solitaire game "Life". Sci. Am. 223 (1970) 120-123.

9. Taylor Charles and Jefferson David: Artificial life as a tool for biological inquiry. In Christopher G. Langton (ed), Artificial Life: an overview, Cambridge, Massachusetts, 1995. MIT Press (1995) 1-14.

10. Ray, T. S.: Artificial Life, In Renato Dulbecco, David Baltimore, François Jacob, Rita Levi-Montalcini (eds), Frontiers of Life, Vol. 1, One The Origins of Life, Academic Press (2001) 107-124.

11. Emmeche, C.: The Garden in the Machine, Princeton (1994).

12. Adami, C.: Introduction to Artificial Life. Springer-Verlag, Berlin (1998).

13. Heins, Y.: Survival and Dormancy of Microorganism, John Wiley \& Sons (1987).

14. Roszak, D. B., and R. R. Colwell.: Survival strategies of bacteria in the natural environments, Am. J. Pub. Health, Vol. 51 (1987) 365-379.

15. Barer, M.R. and Harwood, C.R. (1999) Bacterial viability and culturability, Adv. Microb. Physiol., Vol. 41 (1999) $93-137$.

16. Heudin, J.C. (1998) Virtual Worlds, Proceedings of the First Int. Conf. on Virtual Worlds, Vol. 1434, Springer-Verlag Lecture Notes in Computer Science (Berlin) (1998). 